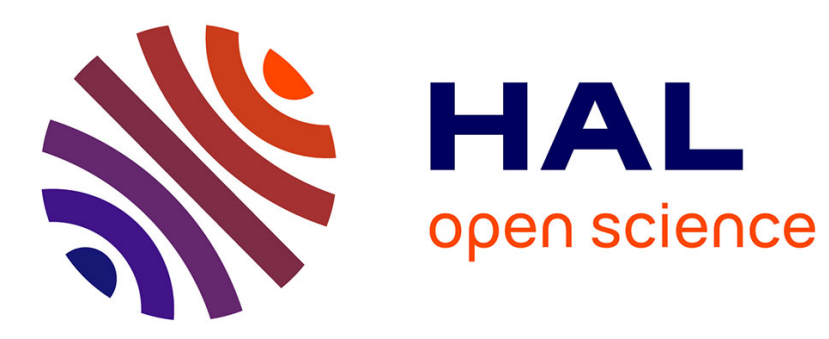

\title{
Exploiting Electric Vehicles Mobility to Reduce Grid Dependency in Transit Areas
}

\author{
Wenjing Shuai, Patrick Maillé, Alexander Pelov
}

\section{To cite this version:}

Wenjing Shuai, Patrick Maillé, Alexander Pelov. Exploiting Electric Vehicles Mobility to Reduce Grid Dependency in Transit Areas. ENERGYCON 2016: IEEE International Energy Conference, Apr 2016, Leuven, Belgium. pp.1 - 6, 10.1109/ENERGYCON.2016.7513999 hal-01375939

\section{HAL Id: hal-01375939 \\ https://hal.science/hal-01375939}

Submitted on 3 Oct 2016

HAL is a multi-disciplinary open access archive for the deposit and dissemination of scientific research documents, whether they are published or not. The documents may come from teaching and research institutions in France or abroad, or from public or private research centers.
L'archive ouverte pluridisciplinaire HAL, est destinée au dépôt et à la diffusion de documents scientifiques de niveau recherche, publiés ou non, émanant des établissements d'enseignement et de recherche français ou étrangers, des laboratoires publics ou privés. 


\title{
Exploiting Electric Vehicles Mobility to Reduce Grid Dependency in Transit Areas
}

\author{
Wenjing Shuai, Patrick Maillé, Alexander Pelov \\ Networks, Security, Multimedia Department \\ Institut Mines-Telecom/Telecom Bretagne \\ Email: \{wenjing.shuai, patrick.maille, alexander.pelov\}@telecom-bretagne.eu
}

\begin{abstract}
The soaring electricity demand due to Electric Vehicles (EVs) increases the urgency of the evolution from the power grid to the so-called smart grid, to manage demand peaks with minimal infrastructure costs.

In this paper, we propose an approach close to Vehicle-toGrid, where EVs can give back some energy from their batteries during peak times. But we also use EVs as energy transporters, by taking their energy where it is consumed. A typical example is a shopping mall with energy needs, benefiting from customers coming and going to alleviate its grid-based consumption, while EV owners make profits by reselling energy bought at off-peak periods.

Based on a simple model for EV mobility, energy storage, and electricity pricing, we quantify the reduction in energy costs for the EV-supported system, and investigate the conditions for this scenario to be viable.
\end{abstract}

\section{INTRODUCTION}

One of the main challenges of the Smart Grid is to manage the enormous increase in electricity needs brought by the arrival and rapid adoption of EVs. But EVs also create opportunities for the grid, as they create a network of mobile energy containers; hence several propositions to use this energy during peak periods-the so-called Vehicle-to-Grid (V2G)-have been issued and studied [1], [2].

$\mathrm{V} 2 \mathrm{G}$ can be implemented in residential areas to reduce the load of transformers [3], [4], to provide ancillary services for the grid [5] or to enhance its capability to face the penetration of renewable energy [6]. Research is quite abundant concerning these possibilities whereas their implementation requires broader cooperation between the grid operator and EV owners. Here, we consider an energy consumer who cannot avoid usage during the peak hours, but fortunately situated in a transit area where EVs stop by frequently.

The literature about managing aggregated EVs is quite abundant, but most works [7]-[10] emphasize on charging EVs only, rather than discharging as we suggest here. In [7], the charging power of the EVs parked is locally optimized, considering their sojourn time. This requires EV owners to inform the controller of their predicted departure time, an assumption also made in [9]. Here we do not rely on such knowledge, since the departure time may be hard to predict by EV owners, who may also be reluctant to disclose it and/or willing to strategically declare it to maximize their benefit. We therefore stick to the simplest case where the facility does not know when EVs will be leaving; that knowledge could nevertheless yield further improvements, which can be studied in future work. Among the approaches that do not require users reporting their departure time, queuing theory can be used to model the dynamics of clients [10], whereas the goal is to serve the most EVs with limited energy for a network of fast charging stations. [8] also applies queuing theory to estimate the waiting time of EVs in a parking lot, and highlight the importance of the number of chargers in the parking lot. Interestingly, measurements shown in that paper illustrate a very good match between the power needs of the facility (a shopping center in the paper) and the arrivals of EVs, motivating further our approach of using EVs-some of which would be willing to sell energy-to reduce the grid-based consumption of the facility.

In this paper, we consider that EV owners sell part of their stored energy directly to an entity with power needs. This has the advantage of avoiding benefit losses due to intermediaries, but also of avoiding energy losses due to transportation. Finally, this comes at no cost in terms of grid management. An illustrative use case is that of a shopping center with energy needs during the day, a time when electricity prices peak but also customers come and go, and we intend to benefit from EVs in the parking lot by installing dischargers for those willing to sell their energy to the shopping center, as illustrated in Figure 1, where the first and second EV are discharging while the third one is not, due to the exhaustion of its surplus energy. When the power discharged from those EVs does not cover its needs, the facility can buy the rest from the power grid. Although our proposition seems opposite to the current practice of putting charging (not discharging) stations in parking lots: we rather think that our approach is complementary; the EV charging stations can either be seen as part of the consumption of the center, or as a separate (since managed from the grid) system. In this paper we consider constant power needs of the center, fitting more with the latter interpretation, but future work can also consider demand variations due to EV charging. More generally, we are interested in a facility where EVs come and go during the day, and with some electricity needs during that period (e.g., a shopping center, a bank, an administrative center... but also a factory that would be located near a shopping center). The facility can buy energy from the grid, at the (relatively high) on-peak price, or from the EVs that are present. 


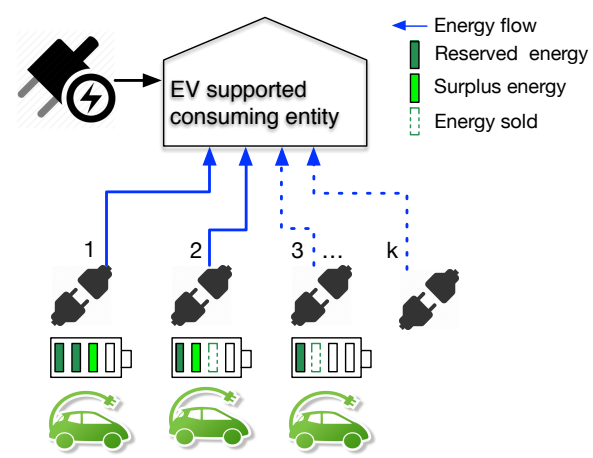

Fig. 1. System implementation: EVs can sell their surplus energy (bought off-peak) to make profit and reduce the facility grid dependency during peaks.

In this paper, we take into account the costs involved with setting such a system, and perform a quantitative analysis of its economic interest. The main exogenous variables are the on-peak and off-peak electricity prices, the mobility of EV owners (arrival rate and sojourn time), the facility needs, and the energy EVs can sell; the decision variables include the number of discharging slots to install and the management of slots occupied by an EV with no more energy to sell. We propose two management schemes to discharge available EVs. For both we carry out an analytical study and show numerical results. Both schemes reduce energy costs for the facility (from $5 \%$ to $15 \%$ ); the difference lying in the tradeoff between less management required (scheme 1) and less discharging stations needed (scheme 2).

The paper is organized as follows. Section II describes our model and the general objectives, while Section III details two management schemes and is emphasized on optimizing the decision variables. Section IV shows the performance of our approaches and the impact of certain parameters. Section V concludes the paper and suggests directions for future work.

\section{MODEL DESCRIPTION}

This section describes the assumptions we make regarding grid electricity prices, electricity needs for the consuming facility (hereafter simply called the facility), and EVs mobility and supply, in order to compute the overall electricity cost for the facility.

Table I summarizes the notations of the model, and specifies the values we consider for the numerical analysis of Section IV.

\section{A. Time-of-use electricity pricing}

We assume in this paper that the grid charges for electricity usage depending on the time of consumption. More specifically, our model considers only two electricity prices: a low price during off-peak periods (typically, at night) and a high price during on-peak periods (during the day). There may be several other prices (e.g., during the day), in which case our model also applies, by just ignoring the other day-time

\footnotetext{
${ }^{1}$ We define "small" in Section III-C
}

TABLE I

MODEL VARIABLES

\begin{tabular}{|c|c|c|}
\hline Var. & Value & Meaning and unit \\
\hline \hline$P_{f}$ & 200 & Facility power needs $(\mathrm{kW})$ \\
\hline$\lambda$ & 10 to 30 & EV arrival rate (hour $\left.{ }^{-1}\right)$ \\
\hline $1 / \mu$ & 1 & Average parking duration (hour) \\
\hline$\eta$ & $88 \%$ & Battery recharging efficiency \\
\hline $1 / \theta$ & 4 to 20 & Average surplus energy per EV $(\mathrm{kWh})$ \\
\hline$\epsilon$ & 0.01 & Discharging inefficiency $\left(\mathrm{kW}^{-1}\right)$ \\
\hline$\beta$ & 1.1 & EV owners resell relative benefit \\
\hline$g$ & 0.25 & On-peak grid electricity price $(€ / \mathrm{kWh})$ \\
\hline$v$ & 0.1 & Off-peak grid electricity price $(€ / \mathrm{kWh})$ \\
\hline$A_{d}$ & 0.2 & Cost per discharger $(€ /$ hour) \\
\hline$U$ & 0.1 & EV unplugging cost in Scheme $2(€)$ \\
\hline$v_{0}$ & $v \beta / \eta$ & EV reselling price $(€ / \mathrm{kWh})$ \\
\hline$k$ & & Number of dischargers in Scheme 1 \\
\hline$k^{\prime}$ & & Number of dischargers in Scheme 2 \\
\hline$P_{0}$ & To optimize & Discharging power per EV $(\mathrm{kW})$ \\
\hline$P_{0}^{*}$ & & Optimal value of $P_{0}$ for small ${ }^{1} k$ \\
\hline$P^{*}$ & $P_{0}^{*}\left(1-\epsilon P_{0}^{*}\right)$ & Obtained power from a discharging EV $(\mathrm{kW})$ \\
\hline$p_{\mathrm{EV}}$ & $v_{0} /\left(1-\epsilon P_{0}\right)$ & EV electricity price seen by facility $(€ / \mathrm{kWh})$ \\
\hline$m_{t}$ & & Current number of parked EVs \\
\hline$n_{t}$ & & Current number of discharging EVs \\
\hline$C_{1}$ & & Total cost bore by facility, Scheme $1(€ / \mathrm{kWh})$ \\
\hline$C_{2}$ & & Total cost bore by facility, Scheme $2(€ / \mathrm{kWh})$ \\
\hline$\rho$ & & EV traffic intensity $\lambda / \mu($ Erlang) \\
\hline$B^{\prime}()$. & & Loss probability of Scheme 1 \\
\hline$B^{\prime}()$. & & Loss probability of Scheme 2 \\
\hline & &
\end{tabular}

prices: what matters is just the current electricity price, and the price paid by EV owners to recharge. However, to optimally decide the number of discharging slots and the management, we would need a specific model of price variations over time, hence the simple choice here of a single day-time price.

Let us denote by $g$ (in monetary units per kWh) the on-peak and by $v<g$ the off-peak price. Note that the latter is the price at which EVs can charge their batteries.

\section{B. Electricity demand of the facility}

We consider a simple model where the facility needs a constant power, denoted by $P_{f}$ (in $\mathrm{kW}$ ), during its activity periods. Without loss of generality we assume that those periods are included within peak-price periods, since during off-peak periods the facility can simply buy energy from the grid.

\section{Potential supply from EVs}

It is unrealistic to assume that EV owners perfectly predict the energy needs of their EVs for the next day, and charge their batteries accordingly. Instead, we think it is reasonable to consider that most EVs carry some surplus energy; when the owners realize during the day that they will not use all of the stored energy, they may choose to sell it to the facility. Because of the variety of battery models and of users distance left to cover within the day, the amount of energy that EVs can provide should be modeled as a random variable. To keep the analysis tractable we consider an exponential distribution, and denote its parameter by $\theta$, so the average surplus energy per EV is $\frac{1}{\theta} \mathrm{kWh}$. 


\section{EV mobility}

We assume that EVs arrive to the parking lot according to a Poisson process with parameter $\lambda$. This process assumption is reasonable, as the result of the uncoordinated behavior of many potential users. We also model the parking duration of each $\mathrm{EV}$ as independent random variables, exponentially distributed with mean $1 / \mu$.

\section{E. Costs faced by the facility}

The facility undergoes several different costs related to its energy consumption, we list them below.

- Grid electricity price. As evoked previously, the facility can buy energy from the grid at a (high) on-peak price $g$ (per kWh)

- EV electricity price. The price paid to EVs should at least compensate the owners' expenses to charge (at the night price $v$ ). Moreover, we assume that the facility provides $10 \%$ of the that expense as an incentive to attract EV owners to joint the discharging program, i.e. they are payed $110 \%$ of the recharging cost, thus a relative benefit $\beta=110 \%$. According to [11] the battery recharging efficiency is over $88 \%$, so conservatively we set $\eta=88 \%$. Discharging efficiency varies between $80 \%$ and $95 \%$ according to [12]. We model this loss through a Joule heating loss proportional to the square of the discharging power, hence equaling $\epsilon P_{0}^{2}$ for some loss factor $\epsilon$. So only the power $P=P_{0}\left(1-\epsilon P_{0}\right)$ is retrieved by the facility if the transfer power is $P_{0}$. In order to fit our setting to the loss values, we set $\epsilon$ so that at low discharging power $\left(P_{0}=5 k W\right), 1-\epsilon P_{0}=0.95$. Thus $\epsilon=0.01$ in our model. We consider that $p_{\mathrm{EV}}$

$$
p_{\mathrm{EV}}:=\frac{v \beta}{\eta\left(1-\epsilon P_{0}\right)}=\frac{v_{0}}{1-\epsilon P_{0}} .
$$

is the actual unit price seen by the facility (the EV actually sees $v_{0}$ ).

- Discharging slots costs. Each discharging slot (discharger) is assumed to cost the facility an amount $A_{d}$ per time unit, hence a trade-off with installing slots to retrieve more energy from EVs.

- Management costs. In addition to those costs, there may be some extra costs in some management solutions, namely, a cost for replacing an EV having sold all of its surplus energy with another one as described thereafter.

\section{F. Management options}

The main idea of our proposition is to reduce the electricity costs by discharging EVs through $k$ dischargers. We will distinguish two possibilities:

- in the "no unplugging" one, no action is carried out when an EV has sold all its energy surplus-that EV occupies the discharger until its departure from the parking lot-;

- the "unplugging" option allows to free a discharger when needed, at a fixed cost $U$, when a new EV enters the system while all dischargers are occupied and at least one by an EV with no more energy to sell.
In the following section, for both management options we calculate the optimal number of dischargers to install to minimize the facility costs, and we compare those costs to the case without the EV discharging option.

\section{ANALYSIS AND COST MINIMIZATION}

In this section, we consider the decision variables of the facility with regard to the discharging system-namely, the discharging power to use and the number of dischargers to install-and analyze their optimal (i.e., cost-minimizing) values for both management schemes. We start with a detailed reasoning under the no-unplugging scheme, followed by a brief discussion of the unplugging scheme.

\section{A. Stochastic analysis}

From the EV mobility model of Section II, the number of EVs parked and plugged to a discharger (which we denote by $m_{t}$ ) is a continuous-time Markov chain, whose evolution is that of an $\mathrm{M} / \mathrm{M} / k / k$ queue, with steady-state distribution

$$
\mathbb{P}_{\text {steady-state }}\left(m_{t}=m\right)=\frac{\rho^{m} / m !}{\sum_{i=0}^{k} \rho^{i} / i !}
$$

where $\rho:=\lambda / \mu$. The loss probability $B(k)$ is then given by the Erlang B formula:

$$
B(k)=\mathbb{P}_{\text {steady-state }}\left(m_{t}=k\right)=\frac{\rho^{k} / k !}{\sum_{i=0}^{k} \rho^{i} / i !} .
$$

Since the sellable surplus energy of each EV is limited, it may not keep discharging before it departs. Therefore not all the $m_{t}$ parked EVs are discharging: only $n_{t}\left(n_{t} \leq m_{t}\right)$ of them are, then the process $\left(n_{t}, m_{t}\right)$ is a continuous-time Markov chain whose transition diagram is depicted in Figure 2, in which $P_{0}$ is decided by the facility.

\section{B. Cost function of the facility}

We observe $n$ EVs discharging simultaneously with probability $\mathbb{P}_{\text {steady-state }}\left(n_{t}=n\right)$ (referring to Section III-A and Figure 2). In that case, the cost imposed on the facility is

$$
\operatorname{Cost}(k, n)=n P_{0} v_{0}+(P_{f}-n \underbrace{P}_{=P_{0}\left(1-\epsilon P_{0}\right)}) g+k A_{d}
$$

In this paper, we assume that the facility is sensitive to its average total cost, i.e., to the time average of the instantaneous cost in (4), that we denote by $C_{1}$ and depends on the number of discharging slots:

$$
C_{1}(k)=\sum_{n=0}^{k} \operatorname{Cost}(k, n) \mathbb{P}_{\text {steady-state }}\left(n_{t}=n\right)
$$

\section{Optimal discharging power with small $k$}

To avoid fast degradation of users' batteries, we try to keep the discharging power per EV at a constant level $P_{0}$ regardless of how many of them are discharging simultaneously. But this may lead to over-supplying, when the number of discharging EVs gets large. So we start by assuming $k \leq \frac{P_{f}}{P_{0}\left(1-\epsilon P_{0}\right)}$, i.e., even when all the $k$ dischargers are withdrawing electricity, 

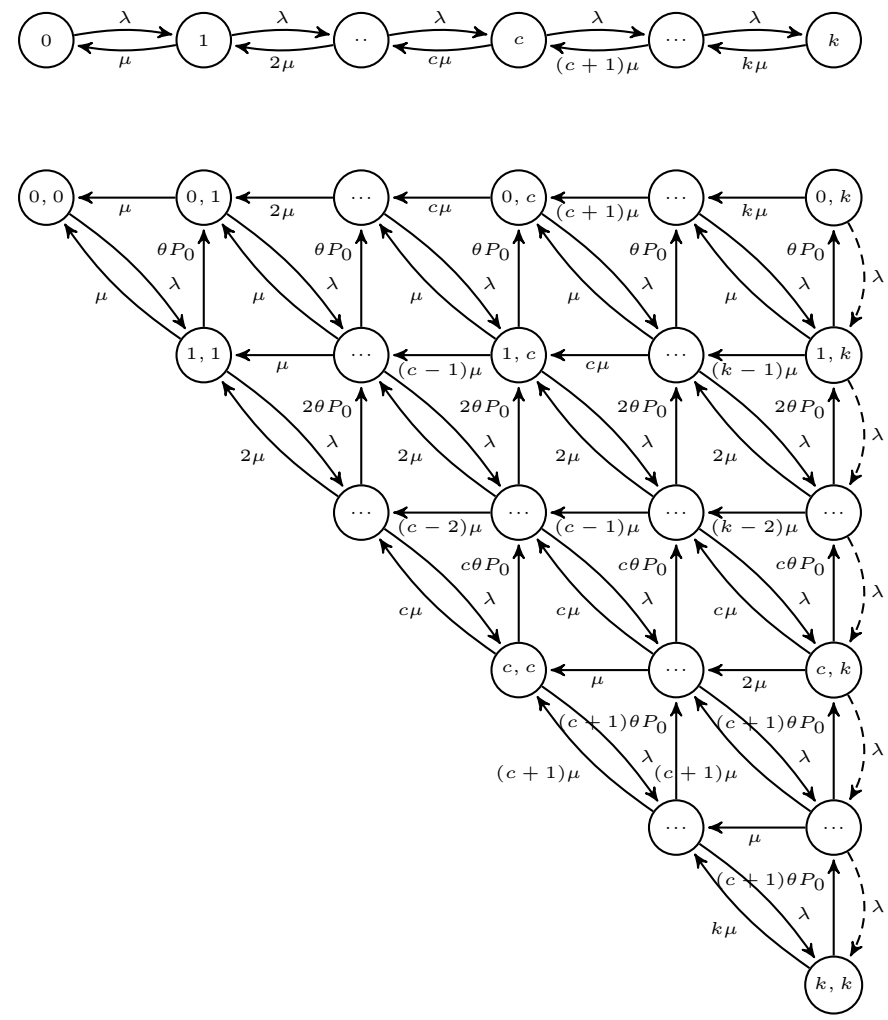

Fig. 2. Continuous-Time Markov Chains describing the evolution of the number of plugged EVs $m_{t}$ (top) and of $\left(n_{t}, m_{t}\right)=($ nb_discharging_EVs,nb_plugged_EVs) (bottom) .

their output together won't exceed the facility needs. For notation simplicity, the number $k$ of charging slots will be called small if $k \leq \frac{P_{f}}{P_{0}\left(1-\epsilon P_{0}\right)}$. For larger values of $k$, we will allow $P_{0}$ to vary with $n_{t}$.

Since we chose $P_{0}$ independent of $n$, the average discharging duration per $\mathrm{EV}$ is $\frac{1}{\mu+\theta P_{0}}$, hence from Little's law the average number of discharging EVs $\bar{N}\left(P_{0}, k\right)$ is $(1-B(k)) \frac{\lambda}{\mu+\theta P_{0}}$. Consequently, the average cost then equals

$$
C_{1}(k)=\bar{N}\left(P_{0}, k\right) P_{0} v_{0}+\left(P_{f}-\bar{N}\left(P_{0}, k\right) P\right) g+k A_{d} .
$$

Differentiating, we obtain the cost-minimizing discharging power $P_{0}^{*}$ as

$$
P_{0}^{*}:=\frac{\mu}{\theta}\left(\sqrt{1+\frac{\theta}{\epsilon \mu}\left(1-\frac{v_{0}}{g}\right)}-1\right) \text {, }
$$

from which the facility actually extracts

$$
P^{*}:=P_{0}^{*}\left(1-\epsilon P_{0}^{*}\right)
$$

per discharging EV. Expression (6) indicates that the discharging power per EV is independent of the power demand of the facility, which is a desirable property: that optimal discharging power would not change even if the facility demand varies over time, as long as $n \leq \frac{P_{f}}{P^{*}}$.

\section{Setting the discharging power}

Recall that the optimal discharging power above is only valid under the assumption $k \leq \frac{P_{f}}{P^{*}}$. For larger values of $k$ (to let in more EVs, and possibly reduce costs further), we keep that power when less than $\frac{P_{f}}{P^{*}}$ cars are discharging. When more than $\frac{P_{f}}{P^{*}}$ discharging cars are discharging, we reduce evenly the power obtained from each EV to exactly cover the whole demand $P_{f}$. Formally, the discharging power from the EV point of view when there are $n$ discharging EVs is then

$$
P_{0}(n)= \begin{cases}P_{0}^{*}=\frac{\mu}{\theta}\left(\sqrt{1+\frac{\theta}{\epsilon \mu}\left(1-\frac{v_{0}}{g}\right)}-1\right) & \text { if } n \leq \frac{P_{f}}{P^{*}} . \\ \frac{1-\sqrt{1-4 \epsilon P_{f} / n}}{2 \epsilon} & \text { otherwise. }\end{cases}
$$

From the facility point of view, the energy extracted from each discharging EV depends on the number $n_{t}$ of discharging EVs, and is summarized as

$$
\min \left(P^{*}, \frac{P_{f}}{n_{t}}\right)
$$

with $P^{*}$ given in (7).

\section{E. Dimensioning the system in the no-unplugging scheme (Scheme 1)}

Given the decision variables, we can compute the average cost per hour given in (5). Due to the complexity of the Markov chain in Figure 2, we do not solve $\mathbb{P}_{\text {steady-state }}\left(n_{t}=n\right)$ analytically, but rather perform an exhaustive search on $k$ to choose the optimal number of discharging slots to install. Numerical results are shown in the next section.

\section{F. Unplugging depleted vehicles when new ones arrive (Scheme 2)}

In the previous scheme, we do not intervene while EVs are parked: this requires less management but also lowers the usage of the dischargers since they can be occupied by depleted EVs and prevent new EVs from entering the system. We therefore introduce an unplugging and replacing procedure in this scheme: when a new EV arrives and all dischargers are occupied, we remove one of the depleted EVs (if any) to make room for that new one, whose surplus energy is alway positive under our assumptions. When compared to the "no unplugging" scheme, this procedure allows new EVs to enter the system as soon as not all dischargers are occupied by discharging EVs (instead of discharging or depleted EVs). In Figure 2, this corresponds to adding the dashed transitions. Actually in that case, the number of discharging EVs is simpler to study, since it is described by a one-dimensional Markov chain, whose transition diagram is given in Figure 3. When the number $k^{\prime}$ of discharging stations is low $\left(k^{\prime} \leq \frac{P_{f}}{P^{*}}\right)$, this corresponds to an $\mathrm{M} / \mathrm{M} / k^{\prime} / k^{\prime}$ queue with stationary distribution

$$
\mathbb{P}_{\text {steady-state }}^{\prime}\left(n_{t}=n\right)=\frac{\rho^{\prime} n / n !}{\sum_{i=0}^{k^{\prime}} \rho^{\prime i} / i !}
$$

with $\rho^{\prime}:=\lambda /\left(\mu+\theta P_{0}^{*}\right)$. In particular, the loss probability (rejection probability for an arriving EV) in that case is 


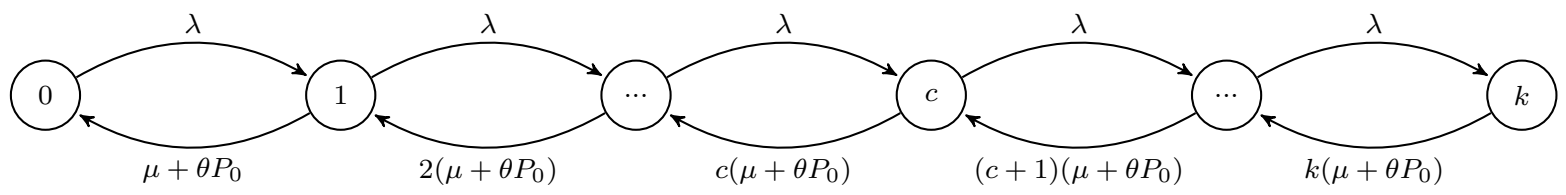

Fig. 3. Transition diagram for the number of discharging EVs in the unplugging scheme (Scheme 2)

$B^{\prime}\left(k^{\prime}\right):=\mathbb{P}_{\text {steady-state }}^{\prime}\left(n_{t}=k^{\prime}\right)$. For larger values of $k^{\prime}$, the steady-state distribution can easily be computed numerically.

Since removing depleted vehicles puts additional management cost to the facility, we now quantify this cost to investigate its impact on the final performance of this scheme. We assume that this cost is linear of the workload, i.e., of the average number of EVs to unplug per time unit. When there are $k^{\prime}$ spots, this workload $W\left(k^{\prime}\right)$ equals the throughput increase from using Scheme 2 instead of Scheme 1, that equals

$$
W\left(k^{\prime}\right)=\lambda\left(B\left(k^{\prime}\right)-B^{\prime}\left(k^{\prime}\right)\right)
$$

Multiplying this workload by a constant unplugging cost per $\mathrm{EV}$, denoted by $U$, gives the additional cost of unplugging depleted EVs that stand in the way of arriving ones. Adding up all costs, the instantaneous cost of Scheme 2 is

$$
\operatorname{Cost}^{\prime}\left(k^{\prime}, n\right)=n P_{0} v_{0}+(P_{f}-n \underbrace{P}_{=P_{0}\left(1-\epsilon P_{0}\right)}) g+k^{\prime} A_{d}+W\left(k^{\prime}\right) U,
$$

and the average cost is then

$$
C_{2}\left(k^{\prime}\right)=\sum_{n=0}^{k^{\prime}} \operatorname{Cost}^{\prime}\left(k^{\prime}, n\right) \mathbb{P}_{\text {steady-state }}^{\prime}\left(n_{t}=n\right) .
$$

Since the partial derivatives of $C_{2}$ on $P_{0}$ have the same form as that of $C_{1}$, we keep using the discharging power $P_{0}$ given in (8). As for Scheme 1, the number $k^{\prime}$ of discharging slots (dischargers) will be computed numerically so as to minimize the average cost $C_{2}\left(k^{\prime}\right)$.

\section{NUMERICAL RESULTS}

This section shows the performance of our proposed schemes in terms of the energy cost they save for the facility, namely $\frac{g P_{f}-C_{1}\left(P_{0}, k\right)}{g P_{f}}$ for the no-unplugging scheme (Scheme 1) and $\frac{g P_{f}-C_{2}\left(P_{0}, k^{\prime}\right)}{g P_{f}}$ for the scheme with unplugging (Scheme 2 ). Note that this relative saving is always nonnegative, since the number of dischargers is optimized to minimize cost; hence in the worst case no dischargers are installer and savings are null.

Free unplugging, as a special case of Scheme 2, is also plotted, to give an idea of the impact of the unplugging cost. The parameter values used for this numerical analysis are shown in Table I. For the value of the off-peak price we use the night price, and for that of the on-peak price we take the peak price for enterprise users, both offered by the French utility company $\mathrm{EDF}^{2}$. .

\footnotetext{
${ }^{2}$ http://entreprises.edf.com/fichiers/fckeditor/Commun/Entreprises/pdf/ 2014/BAREME_TARIF_VERT_01_11_2014.pdf
}

\section{A. How many dischargers to install?}

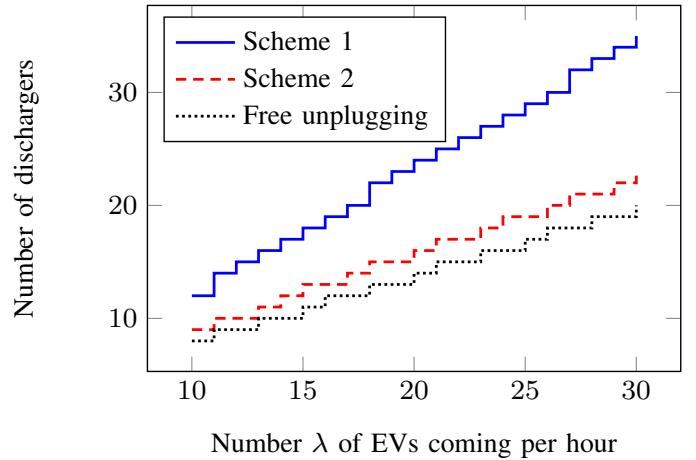

Fig. 4. Optimal number of dischargers according to EV arrival rate $\lambda$

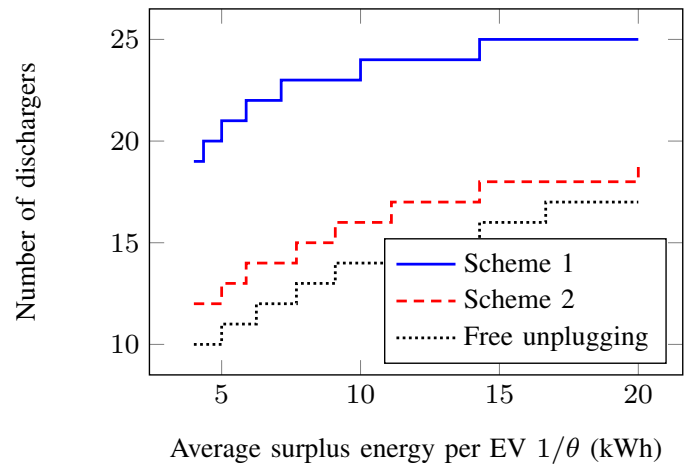

Fig. 5. Optimal number of dischargers versus average surplus energy $1 / \theta$

Figures 4 and 5 show the optimal number of discharging stations to install for each scheme: interestingly, Scheme 2 needs much fewer dischargers than Scheme 1. And as the "free unplugging" benchmark shows, the unplugging cost has a direct effect on the optimal number of dischargers, conform to intuition (the higher the cost, the more dischargers to avoid unplugging situations). These optimal numbers of dischargers are applied in the following two subsections, which compare the performance of the two schemes.

\section{B. The difference between rush hour and vacant hour}

It is not surprising to find that the more EVs come in a unit time (i.e., the larger $\lambda$ is), the more saving they bring to the facility, as illustrated in Figure 6. The average surplus on-board energy $1 / \theta$ is fixed at $10 \mathrm{kWh}$, and the average time interval between two EVs' coming decreases from $6 \mathrm{~min}$ to $1.5 \mathrm{~min}$. The gap between "Scheme 1" and "Scheme 2" increases since 


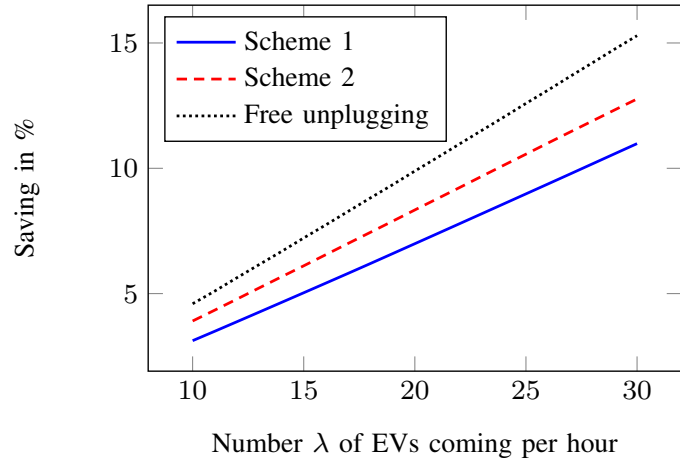

Fig. 6. Variation of saving according to the EV arrival rate $\lambda$

higher coming rate results in more dischargers occupied by depleted EVs upon new arrivals, which is also causing the increase of the gap between curve "Scheme 2" and that of "Free unplugging".

\section{Effect of the surplus energy in EVs}

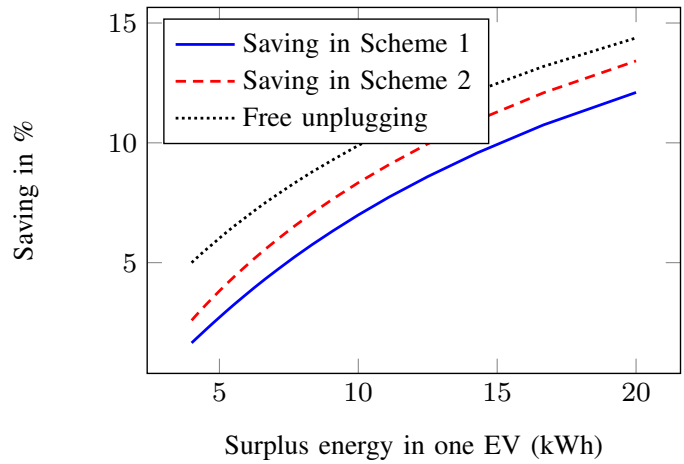

Fig. 7. Variation of saving versus average EV surplus energy $1 / \theta$

Another key parameter is the available energy onboard parked EVs, whose average is denoted by $1 / \theta$ in our model. According to a survey [13], more than $90 \%$ of daily travel is less than 100 miles, which consumes $34 \mathrm{kWh}$ of energy for a Tesla Model S whose battery capacity is $85 \mathrm{kWh}$, or $15 \mathrm{kWh}$ for a compact car whose battery capacity is typically $21 \mathrm{kWh}$ [14]. So we range the average surplus energy from $4 \mathrm{kWh}$ to $20 \mathrm{kWh}$, hence the probability of having an EV with more than $50 \mathrm{kWh}$ of sellable energy is between $0.0018 \%$ and $0.13 \%$, which is very rare. So our system doesn't require unrealistically large battery size, even less than $10 \mathrm{kWh}$ of average surplus energy per EV can significantly reduce the facility electricity bill for the parameters we consider.

\section{CONCLUSION}

In this paper we propose to use the surplus energy in EVs gathered in a parking lot to support the energy needs of a facility: the mobility of EVs during the day brings energy on a quite regular basis, to an extend that possibly largely exceeds what could be stored in a unique (even large) battery that would be controlled by the facility. Leveraging the storage capabilities of EV batteries, our scheme benefits both

- EV owners, who can sell during peak times some energy bought during off-peak period and make some profit;

- and the facility, which can benefit from energy at lowerthan-peak price, without installing large storage solutions.

We propose and evaluate two management schemes to discharge those EVs, namely without and with the possibility of removing depleted ones. Our numerical results suggest that we can save around $10 \%$ on the energy bill, and we don't need large amounts of surplus energy in each EV to realize that. Hence this approach is viable in our opinion, and could help reduce demand peaks that are observed in nowadays grids.

Possible extensions of our work include considering an elastic EV supply, by relating the proportion of users who agree to discharge their batteries to the incentive $\beta$ provided by the facility, and then searching for the optimal incentives. Also, selecting upon arrivals EVs with sufficient sellable energy could significantly reduce the unplugging workload.

\section{REFERENCES}

[1] S. Letendre and W. Kempton, "The V2G concept: A new model for power?" Public Utilities Fortnightly, vol. 140, no. 4, pp. 16-26, Feb. 2002.

[2] J. Pillai and B. Bak-Jensen, "Integration of Vehicle-to-Grid in the western Danish power system," IEEE Trans. Sustain. Energy, vol. 2, no. 1, pp. 12-19, 2011.

[3] H. Liang, B. J. Choi, W. Zhuang, and X. Shen, "Towards optimal energy store-carry-and-deliver for PHEVs via V2G system," in Proc. IEEE INFOCOM, 2012, pp. 1674-1682.

[4] — - "Optimizing the energy delivery via V2G systems based on stochastic inventory theory," IEEE Trans. Smart Grid, vol. 4, no. 4, pp. 2230-2243, Dec 2013.

[5] S. Sun, M. Dong, and B. Liang, "Real-time welfare-maximizing regulation allocation in dynamic aggregator-EVs system," IEEE Trans. Smart Grid, vol. 5, no. 3, pp. 1397-1409, May 2014.

[6] U. Chukwu and S. Mahajan, "V2G parking lot with PV rooftop for capacity enhancement of a distribution system," IEEE Transactions on Sustainable Energy, vol. 5, no. 1, pp. 119-127, Jan 2014.

[7] Y. He, B. Venkatesh, and L. Guan, "Optimal scheduling for charging and discharging of electric vehicles," IEEE Transactions on Smart Grid, vol. 3, no. 3, pp. 1095-1105, Sept 2012.

[8] Q. Gong, S. Midlam-Mohler, E. Serra, V. Marano, and G. Rizzoni, "PEV charging control for a parking lot based on queuing theory," in American Control Conference (ACC), June 2013, pp. 1124-1129.

[9] E. Akhavan-Rezai, M. Shaaban, E. El-Saadany, and F. Karray, "Prioritybased charging coordination of plug-in electric vehicles in smart parking lots," in IEEE PES General Meeting - Conference Exposition, July 2014, pp. 1-5.

[10] I. Bayram, G. Michailidis, M. Devetsikiotis, and F. Granelli, "Electric power allocation in a network of fast charging stations," IEEE Journal on Selected Areas in Communications, vol. 31, no. 7, pp. 1235-1246, July 2013.

[11] S. Deilami, A. Masoum, P. Moses, and M. Masoum, "Real-time coordination of plug-in electric vehicle charging in smart grids to minimize power losses and improve voltage profile," IEEE Transactions on Smart Grid, vol. 2, no. 3, pp. 456-467, Sept 2011.

[12] N. Bizon, "Energy efficiency of multiport power converters used in plugin/V2G fuel cell vehicles," Applied Energy, vol. 96, no. 0, pp. 431 - 443, 2012, smart Grids.

[13] J. Krumm, "How people use their vehicles: Statistics from the 2009 national household travel survey," SAE, Tech. Rep. 2012-01-0489, April 2012.

[14] C. Guenther, B. Schott, W. Hennings, P. Waldowski, and M. A. Danzer, "Model-based investigation of electric vehicle battery aging by means ofvehicle-to-grid scenario simulations," Journal of Power Sources, vol. 239, no. 0, pp. $604-610,2013$. 